\title{
Epideixis und Encyklopaedie
}

\section{Schlegels Philologie zwischen Kulturwissenschaft und Kulturpolitik}

Eine Philologie, die ihre Arbeit als eine politische versteht, beruft sich gerne auf Friedrich Schlegel. Ein jüngstes Beispiel dafür ist Werner Hamachers wortgewaltige Intervention Für-die Philologie, die (vermittelt durch Walter Benjamins Begriff der Kunstkritik in der deutschen Romantik) Schlegels Überlegungen zur Philologie sogar für eine "Philo-Polemo-Logie« in Stellung bringt. ${ }^{I}$ Ebenso entschieden gibt sich Paul de Man, wenn er die "Rückkehr zur Philologie" gerade deshalb einfordert, weil diese - und zwar in sich selbst - eine "subversive Kraft" entfesseln soll, die politischtheologische Vorurteile desavouiert. ${ }^{2}$ Dafür brauche es keinen »kritischen Terrorismus«, es reiche »eine sensible Gelehrsamkeit«. ${ }^{3}$ Bekanntlich ist es derselbe Paul de Man, der auf den frühen Schlegel zurückgreift, um die für seine eigene 'gelehrsamer Lektürepraxis zentrale Vorstellung einer sich ironisch brechenden Allegorie darzulegen. ${ }^{4}$

I Werner Hamacher: »Für - die Philologie«. In: Jürgen Paul Schwindt (Hg.): Was ist eine philologische Frage? Beiträge zur Erkundung einer theoretischen Einstellung. Frankfurt a. M. 2009, S. 2I-60, hier S. 34. Spätestens in der anschließenden Char-Lektüre wird unmissverständlich deutlich, dass der 'polemischer Aspekt der Philologie auch politische Konflikte im engsten Sinne mitbetrifft. Vgl. ebd., S. 40-44.

2 Der Text erschien erstmals 1982 im Times Literary Supplement. Eine deutsche Übersetzung ist jetzt abgedruckt als Paul de Man: „Die Rückkehr zur Philologie«. In: Kai Bremer/Uwe Wirth (Hg.): Texte zur modernen Philologie. Stuttgart 20I0, S. 216-226, hier S. 220.

3 Ebd., S. 221.

4 So in Paul de Man: "The Concept of Irony«. In: ders.: Aesthetic Ideology. Hg. von Andrzej Warminski. Minneapolis/London 1996, S. I63-I84. David Wellbery geht sogar davon aus, »daß Schlegels Denken für die Ausbildung 
Dass Schlegel für eine solchermaßen politisierte Philologie ins Spiel gebracht werden kann, führt sich auf seine Hefte Zur Philologie zurück, in denen die Philologie als ein gesamtgesellschaftlich emanzipatorisches Projekt profiliert wird. ${ }^{5}$ Die Öffnung zur Gesellschaft hin geht dabei Hand in Hand mit einer gleichsam kulturwissenschaftlichen Ausweitung des Gegenstandsbereichs. Philologie soll folglich das, was seit (und aufgrund) der Romantik 'Kultur heißt, in einen politisch revolutionären Dienst stellen. Wegen dieser Verschränkung von (Kultur-)Politik und Kulturwissenschaft im Zeichen der Philologie werden Figuren wie de Man und Hamacher an Schlegel anknüpfen.

In der Forschung dürfte inzwischen Einigkeit darüber herrschen, dass hier auch und vor allem das gemeinsame Interesse an einem ausgeweiteten Rhetorikbegriff eine Rolle spielt. ${ }^{6}$ Was bislang indessen nicht zureichend scharf gestellt wurde, ist das epideiktische Erbe einer derartigen Rhetorizität. ${ }^{7}$ Dabei ist weniger von Interesse, dass das Verhältnis der Philologie zu den panegyrischen Intentionen des genos epideiktikon erklären ließe, warum - wie Walter Benjamin schon betont hat - alle romantische Kritik eine positive Würdigung sein muss. ${ }^{8}$ Weitaus wichtiger ist es zu sehen,

der de Manschen Romantikkonzeption eine geradezu architektonische Funktion ausübte« (David Wellbery: „Rhetorik und Literatur. Anmerkungen zur poetologischen Begriffsbildung bei Friedrich Schlegel«. In: Ernst Behler/Jochen Hörisch [Hg.]: Die Aktualität der Frühromantik. Paderborn u. a. 1987, S. 16I-173, hier S. 162).

5 Zum Bezug Schlegels zur Philologie siehe jetzt auch Ulrich Breuer/Remigius Bunia/Armin Erlinghagen (Hg.): Friedrich Schlegel und die Philologie. Paderborn u. a. 2013.

6 Spätestens seit Peter Schnyders Monographie kann an der Rolle der Rhetorik für den jungen Schlegel kein Zweifel mehr bestehen. Siehe Peter Schnyder: Die Magie der Rhetorik. Poesie, Philosophie und Politik in Friedrich Schlegels Frühwerk. Paderborn u. a. 1999.

7 Die zentrale Bedeutung der Epideiktik für das Rhetorikverständnis des jungen Schlegel wird zumindest angedeutet von Thomas Schirren: »Rhetorik und Romantik. Schlegels Philosophie der Prosa in den frühen Notaten«. In: Neue Beiträge zur Germanistik (Doitsu Bungaku I45) II (2013), S. I8-46.

8 Walter Benjamin: Der Begriff der Kunstkritik in der deutschen Romantik. In: ders.: Gesammelte Schriften. Hg. von Rolf Tiedemann u. Hermann Schweppenhäuser. Bd. I. Frankfurt a. M. I99I, S. 7-I22, hier S. 67. 

verdeutlichen soll, die, wenn man de Man und Hamacher folgen will, philologischer Forschung (spätestens seit Schlegel) in sich selbst inhäriert. Eine solche philologische Forschung fußt, wie ich im Folgenden zeigen werde, auf einer Selbstaffektion, die niemals rein sein kann und deshalb - um Hamacher zu zitieren - immer auch eine »Heteroaffektion « involviert. ${ }^{9}$ Diese, wenn man so will, sepideiktischer Selbstaffektion erlaubt es Schlegel, die Kreisschlüsse zu sprengen, denen die Argumentationen des post-Kantischen Idealismus und der post-Schleiermacher'schen Hermeneutik gleichermaßen vertrauen, und den enzyklopädischen (en-kyklos) Anspruch beider neu auszurichten. Diese Neuausrichtung ist es, durch die Schlegel auf Philologen wie de Man und Hamacher wirkt.

Die Tragweite dieser Zusammenhänge, die abschließend auch die Frage nach der sogenannten spolitischen Romantikı auf den Plan rufen wird, soll vor dem Hintergrund einer genauen Kantund Fichte-Lektüre verdeutlicht werden. Zuvor jedoch noch einige einleitende Worte zu der Beziehung von Epideiktik und Philologie bei Schlegel selbst.

I. Epideiktische Rhetorik und philosophische Philologie

Den Zusammenhang von (Kultur-)Politik und (kulturwissenschaftlicher) Philologie stiftet Schlegel durch die Übertragung einer rhetorisch-literarischen Strategie in philologische und philosophische Kontexte. Die "Idee einer Philosophie der Philologie« beruht in folgendem Befund: "< die $\phi \lambda[$ Philologie] behandelt alles als Studien und als $\varepsilon \pi \imath \delta \varepsilon \varepsilon_{\xi} \iota^{*}$. $>$ «" Hier ist zweierlei gesagt: Erstens wird der Gegenstandsbereich der Philologie ins Unbestimmte ausgeweitet ("alles«), zweitens wird philologische Arbeit mit dem genos epideiktikon, lateinisch: genus demonstrativum - derjenigen rhetorischen Rede also, die das Gegenwärtige ‘aufzeigt` und damit feiert - in

9 Hamacher: »Für - die Philologie« (s. Anm. I), S. 4I.

Io Friedrich Schlegel: Zur Philologie I. In: KFSA 16, S. 33-56, hier S. 43, Nr. 99. 
Zusammenhang gebracht. Damit ist die Redeform bestimmt, in der Schlegel die Philologie sich äußern sieht: "<Unsere ganze $\phi \lambda\left[\right.$ Philologie] ist epideiktisch und panegyrisch [...].> $"{ }^{\mathrm{II}}$ Diese Worte markieren Einsatzpunkt und Ergebnis von Schlegels Philosophie der Philologie, die sich, an Kant geschult, als Kritik der Philologie, oder genauer: als Kritik eines transzendental-philologischen Scheins vollzieht. Eine solche Kritik, die die Bedingungen der Möglichkeit der Philologie zu bestimmen sucht, muss sich - in einer ebenfalls gut kantisch-reflexiven Geste - selbst der von ihr herausgearbeiteten Möglichkeitsbedingnis unterwerfen. Anders als bei Kant jedoch organisiert sich die Kritik nicht um den philosophischen Schlüsselbegriff der transzendentalen Apperzeption, sondern um den rhetorischen Begriff der epideixis.

Die epideixis ist eine der drei von Aristoteles identifizierten Formen rhetorischer Rede. ${ }^{\mathrm{I} 2}$ Die aristotelische Einteilung reflektiert die unterschiedlichen Anlässe, auf die Rhetorik reagiert. Das Kriterium ihrer Unterscheidung ist die Zeit. Während sich die juristische Rede (dikanikon) mit vergangener Schuld und die politische Rede (symbouleutikon) mit zukünftigen Entscheidungen beschäftigt, widmet sich das epideiktikon der Gegenwart einer nicht nur moralisierten, sondern zugleich auch politisierten Schönheit. ${ }^{13}$ Damit gerät die Rhetorik in die Nähe zur Gelegenheitsdichtung, die im Ausgang von konkreten Anlässen ein dichterisches Lob (aber auch dichterische Kritik) formuliert. Seit der Augusteischen Zeit - etwa in der Ars poetica des Horaz oder in De compositione verborum des Dionysios von Halikarnassos - wird das Band, das in der Praxis zwischen rednerischer Epideiktik einerseits und dichterischer Panegyrik und Paränese andererseits von jeher geknüpft war, auch von der rhetorischen und poetischen Theorie eingeholt. ${ }^{\mathrm{I}}$

Siche zum Folgenden

I2 Siehe zum Folgenden Aristoteles: Ars rhetorica. Hg. von W. Ross. Oxford 1975, 1356b-1359a.

I3 Die griechische kalokagathia war als (Selbst-)Charakterisierung der aristoi unmittelbar auch politisch kodiert.

I4 Für eine konzise Darstellung der sich in der Antike herausbildenden Komplizenschaft von Rhetorik und Dichtung, die die Entwicklung des Begriffs von Literatur maßgeblich begünstigt hat, siehe Roland Barthes, "L'ancienne 
Doch auch schon lange vor Horaz hat Dichtung eine gesellschaftliche Funktion, die auf den Begriff der epideixis gebracht werden kann. In den Siegesliedern eines Pindar oder Bakchylides etwa werden Olympioniken in der Öffentlichkeit der Polis besungen, indem ihre Taten in einen größeren historisch-mythischen Kontext eingebettet werden. Der Sinn, der dadurch erzeugt wird, hat eine unmittelbar gesellschaftspolitische Dimension, denn die Siegeslieder sollen es der Polis ermöglichen, sich ihrer selbst in der Heldenfeier zu versichern. Dass hier insbesondere auf die Tradition der Ode zu verweisen ist, hat allerdings noch einen gewichtigeren Grund als den, die epideiktischen Züge vormoderner Dichtung an ihr zu veranschaulichen. Schließlich wird Schlegel den in ihrer Nachfolge stehenden Klopstock herausstreichen, weil

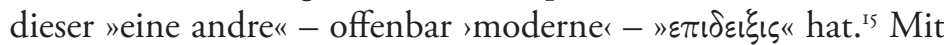
Klopstock, der dem frühen wie dem späten Schlegel als "Stifter und Vater ${ }^{16}$ der neueren deutschen Literatur gilt, emanzipiert sich die Dichtung von der epideiktischen Panegyrik und Paränetik eines höfisch-aristokratischen Kontexts, setzt sich aber nicht als ein autonomes soziales System, das sich nur in und um sich selbst dreht. Vielmehr stellt Dichtung ihre dadurch erlangte Popularität in den Dienst einer politisch revolutionären epideixis, die sich der Menschheit überhaupt weihen will. ${ }^{17}$ Und in der Tat war Klop-

rhétorique. Aide-mémoire«. In: Communications I6 (1970), S. 172-223, hier S. $175-183$.

I5 Schlegel: Zur Philologie I (s. Anm. Io), S. 55, Nr. 234.

I6 So in Friedrich Schlegel: Über das Studium der Griechischen Poesie. In: KFSA I, S. 217-367, hier S. 365. Noch in seiner Wiener Zeit heißt es, Klopstock sei "der Stifter einer ganz neuen Epoche [...] und der eigentliche Meister und Vater der jetzigen deutschen Literatur« (Friedrich Schlegel: Geschichte der alten und neuen Literatur. In: KFSA 6, S. 36I).

I7 Diese Tendenz zeigt sich vor allem in der Revolutionslyrik Klopstocks, etwa in der Ode Der Freyheitskrieg, mit der er den Oberbefehlshaber der Koalitionsarmee von einem Angriff auf die Französische Republik abbringen wollte. Freilich zeigt dieses Gedicht auch, dass Klopstocks Anspruch, für die Menschheit zu sprechen, in einem spannungsvollen Verhältnis zu den nationalistischen Tönen steht, die er ebenfalls anschlägt. Siehe Friedrich Klopstock: Der Freyheitskrieg. In: ders.: Werke und Briefe. Historisch-Kritische Ausgabe. Hg. von Horst Gronemeyer u. a. Bd. I. Berlin/New York 20IO, S. $472-473$. 
stock zu Lebzeiten, und zwar in gebildeten wie in ungebildeten Kreisen, immens populär.

Damit macht sich Klopstock dichterisch Möglichkeiten zunutze, die der Rhetorik der Epideiktik innewohnen. Denn anders als die juristische und politische Rhetorik ist das genos epideiktikon institutionell wenig gebunden. ${ }^{18}$ Dies hat ihm auch vielfach Kritik eingebracht, weil es als politisch opportunistisch und ästhetisch inferior galt. Dass Schlegel hingegen den okkasionellen Charakter dieser Dichtungstradition nicht eo ipso als Einwand gegen ihre literarische Qualität gelten lässt, zeigt sich in den Athenaeums-Fragmenten, wo es heißt: "Daß es den Adel vaterländischer Festgesänge nicht entweihen kann, wenn sie tüchtig bezahlt werden, beweisen die Griechen und Pindar. «" ${ }^{19}$ Popularität muss für Schlegel nicht notwendig mit ästhetischen Kompromissen erkauft werden.

Ferner erlaubt es die Freiheit von institutionellen Kontexten der Epideiktik nicht nur in den Begriff der Literatur einzuwandern, sondern auch eine zentrale Rolle in der sogenannten Anthropologisierung der Rhetorik um I8oo zu spielen. Wie Rüdiger Campe gezeigt hat, verdankt sich diese Anthropologisierung der Rhetorik vornehmlich dem Begriff und der Praxis der Selbstaffektion. ${ }^{20} \mathrm{Um}$ Affekte bei anderen hervorzurufen, lässt sich der Redner erst selbst durch den Anlass seiner Rede affizieren. Zwar führt Campe diese Selbstaffizierung über Quintilian auf die römische Gerichtspraxis zurück (wo der Advokat an Stelle seines Mandanten sprach), sie ist aber mindestens ebenso prominent im Selbstverständnis der

I8 Auf die "fehlende institutionelle Basis« der Epideiktik, auf die daraus folgende "Rhetorikverachtung« sowie auf ihre Nähe zur "Literatur» hingewiesen hat etwa schon Helmut Schanze: „Festrede und Festspiel. Konstellationen und Widersprüche«. In: Josef Kopperschmidt/ders. (Hg.): Fest und Festrhetorik. Zu Theorie, Geschichte und Praxis der Epideiktik. München I999, S. 345-35I, hier S. 345 .

I9 Friedrich Schlegel: Athenäums-Fragmente. In: KFSA 2, S. 165-255, hier S. I8I, Nr. II5.

20 Siehe Rüdiger Campe: „Affizieren und Selbstaffizieren. Rhetorisch-anthropologische Näherung ausgehend von Quintilian Institutio oratoria VI I-2«. In: Josef Kopperschmidt (Hg.): Rhetorische Anthropologie. Studien zum Homo rhetoricus. München 2000, S. 135-152. 
Literatur. In der Ars poetica heißt es hierzu: »se vis me flere, dolendum est / primum ipsi tibi: tum tua me infortunatia laedent" (»Willst du mich zu Tränen nötigen, so mußt du selbst zuvor das Leid empfinden; nur dann wird mich dein Unglück rühren«). ${ }^{21}$

Weil Schlegel ebenfalls an der Popularität einer von höfischen Kontexten emanzipierten Epideiktik teilhaben - und sich dafür vor allem des befreiten Potenzials der Selbstaffizierung bedienen - will, überträgt er das rhetorisch-dichterische Register einer gesellschaftspolitischen epideixis auf die Philologie. Damit erreicht er nicht nur die Annäherung von literarischem und theoretischem Diskurs, die bekanntlich eines der Hauptanliegen der AthenaeumsFragmente ist. In den Heften Zur Philologie heißt es hierzu spezifischer, der »Philolog « müsse »Poet seyn « ${ }^{22}$, der »vollendete Poet « ${ }^{23}$

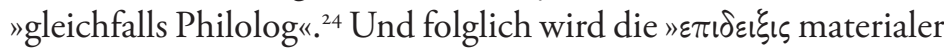
Altertumslehre ${ }^{25}$ in letzter Konsequenz zum $»$ PHILOLOGISCHE[N] Roman. «" Damit kann Schlegel aber darüber hinaus auch die institutionsunabhängigen Energien epideiktischer Rhetorik in den Dienst einer neuen, nicht-institutionalisierten Philologie stellen, die potenziell die gesamte Menschheit (auf eine Weise, die noch genauer zu bestimmen sein wird) umfassen soll. So versucht Schlegel, die Philologie als Vollzugsform dessen zu mobilisieren, was er später als »Transzendentalpoesie ${ }^{27}$ bezeichnen wird.

Insofern eine popularisierte Philologie eine gesamtgesellschaftliche Dynamik entfesseln kann, eignet ihrer Anverwandlung der epideixis auch eine kulturelle wie politische Stoßkraft. Diese wird theoretisch unterfangen von einer Neudeutung des Zirkels im

Quintus Horatius Flaccus: De arte poetica. In: Sämtliche Werke. Hg. von Hans Färber u. Wilhelm Schöne. München 1993, S. 538-575, hier S. 546 f. [V. IO2 f.].

22 Schlegel: Zur Philologie I (s. Anm. Io), S. 49, Nr. I68.

23 Ebd., S. 45, Nr. I28.

24 Ebd., S. 45, Nr. I26.

25 Ebd., S. 48, Nr. 156.

26 Ebd., S. 54, Nr. 220.

27 Schlegel: Athenäums-Fragmente (s. Anm. 19), S. 204, Nr. 238. Bemerkenswerterweise soll dabei Pindar eine Schlüsselrolle spielen: die »nicht seltnen transzendentalen Materialien und Vorübungen « müssten sich, so wird gefordert, "mit der künstlerischen Reflexion und schönen Selbstbespiegelung, die sich« unter anderem auch »bei Pindar« findet, "vereinigen« lassen. 
130 Beweis, die die Anlassgebundenheit philologischer Forschung eigens in Anschlag gebracht hat. Wenngleich diese theoretische Neubestimmung auch Implikationen für Schlegels Hermeneutikverständnis hat, wird sie vornehmlich im Rückgriff auf Kant und in Absetzung von Fichte formuliert.

\section{Cyklisazions-Strategien bei Fichte, Kant und Schlegel}

Eine "Kritik der Philologie« im Sinne Kants hält Schlegel für geboten, weil diese - wie zum Abschluss des ersten Hefts Zur Philologie festgehalten wird - in eine ganze Reihe von "Antinomien« führe, ${ }^{28}$ allen zuvor die über »den Primat der Kritik oder der Hermeneutik «. ${ }^{29}$ Denn Texte sollen, wie im Folgeheft zu lesen steht, »erst berichtigt und dann erklärt werden und umgekehrt. $\aleph^{30}$ Der hermeneutische Kreisschluss wird also mit Rekurs auf die philosophische Sprache Kants behandelt. Schlegels Antinomien gehören jedoch keinem transzendentalen Schein an, der durch den Vernunftgebrauch (sei er nun theoretisch oder praktisch) erzeugt wird, sondern einem Schein, mit dem sich das Fällen von Urteilen konfrontiert sieht. Wenn Philologie, wie Schlegel behauptet, die »kritische Anlage des Menschen kunstmäßig ausbildet «, ${ }^{31}$ dann weil sie wesentlich "Urtheilskunst « ist, die »Urtheilsgenie « fordert, ${ }^{32}$ sich mithin nicht von vorgefassten Begriffen leiten lässt.

Obwohl er von Verstand auf Urteil und von Begriffen aufTexte umstellt, folgt Schlegel dem von der Kritik der reinen Vernunft vorgegebenen Ansatz, wenn er eine "Dedukzion «33 von Philologemen aus philologischen Urteilen fordert. Der in solchem Vorgehen inhärente Kreisschluss wird in einer "cyklische $[\mathrm{n}]$ Methode ${ }^{34}$ programmatisch verankert. Das heißt dann aber auch, dass Schlegel

28 Zur Philologie I (s. Anm. Io), S. 56, Nr. 239.

29 Ebd., S. 55, Nr. 236.

30 Friedrich Schlegel: Zur Philologie II. In: KFSA 16, S. 57-82, hier S. 63, Nr. 39.

3 I Ebd., S. 64, Nr. 5I.

32 Ebd., S. 65, Nr. 53.

33 Ebd., S. 59, Nr. 8.

34 Ebd., S. 66, Nr. 6I. 
Denken unterstellt hatte und mit seiner kritischen Philosophie überwunden haben wollte, ${ }^{36}$ wieder stark machen muss, weil er zwar "so cyklisierend wie möglich" vorgehen will, seine Kritik aber vom Ansatz her »nicht « - und zwar prinzipiell nicht - „cykliziert « sein kann. ${ }^{37}$ Dies liegt nicht etwa daran, dass sich der Kreis wie bei Fichte nur in Annäherung an einen Grenzwert schließen könnte. Schlegel stellt sich hier sogar nachdrücklich gegen "Fichte's doch nicht ganz ins Klare gekommene Cyklisazion «..$^{88}$

Dadurch, dass sich Schlegels Kritik der Philologie als Gegenmodell zum Idealismus eines sich selbst setzenden Ichs versteht, verwandelt sich auch der Charakter der notwendigen Zirkelhaftigkeit philosophischer Forschung von Grund auf. Damit zieht Schlegel die Konsequenzen aus seiner Auseinandersetzung mit Fichtes Wissenschaftslehre, wie sie sich in den sogenannten Philosophischen Fragmenten von 1796 artikuliert. Die fundamentalen Sätze der Philosophie verdanken sich, wie Fichte zu Beginn der Grundlage der gesamten Wissenschaftslehre (1794) einräumt, einem "Zirkel« im Beweis, der "unvermeidlich« ist. ${ }^{39}$ Der dadurch "aufgegebne Kreisgang « kann aber erst im »einst « einer sich ständig entziehenden Zukunft vollendet werden ${ }^{40}$ sodass sich der Kreis nur in der Unendlichkeit schließt. Dem Menschen ist folglich eine perennierende Aufgabe gesetzt, deren Erfüllung an einem Grenzwert angesiedelt ist. Diese Vorstellung eines Kreises, der sich in einer unmöglichen und systemimmanent zugleich notwendigen Unendlichkeit schließen würde, wird von Schlegel in einer solchen Weise zersetzt, dass sich der Kreis an jedem möglichen Segment auf eine Vielzahl sich streuender (und wiederum an jedem möglichen Segment offener) Kreise öffnet. Das Bindungsglied bildet dabei

Ebd., S. 79, Nr. 203.

36 Immanuel Kant: Kritik der reinen Vernunft. Hg. von Jens Timmermann. Hamburg 1998, B 106 f.

37 Schlegel: Zur Philologie II (s. Anm. 30), S. 80, Nr. 2 IO.

38 Ebd., S. 66, Nr. 6I.

39 Johann Gottlieb Fichte: Grundlage der gesamten Wissenschaftslehre. In: ders.: Johann Gottlieb-Fichte-Gesamtausgabe. Hg. von Reinhard Lauth u. Hans Jacob. Bd. I.2. Stuttgart/Bad-Cannstatt 1969, S. 249-45I, hier S. 25 I f.

40 Ebd., S. 420. 
132 das, was in Anschluss an die klassische Rhetorik und an Kant als Anlass bezeichnet werden kann.

So machen die Hefte Zur Philologie einen Aspekt der kreishaften Strukturen von Selbstbezüglichkeit stark, der vor allem in der dritten Kantischen Kritik zum Stolperstein wird (und auf den Schlegels Gebrauch des epideixis-Begriffs aufmerksam macht): die Anlassgebundenheit einer jeglichen Vorstellung. Die Einsicht, dass ein jeder Denkinhalt durch etwas angestoßen wird, das von ihm nicht eingeholt werden kann, erlaubt es Schlegel, den Kreisgang Fichtes auf eine markante Weise zu öffnen. Zu Beginn der Kritik der Urteilskraft macht Kant auf die fundamentale Bedeutung des "Anlasses einer gegebenen Vorstellung " aufmerksam. ${ }^{4 \mathrm{I}}$ Obwohl diese Worte en passant gesagt zu sein scheinen, liegt auf ihnen ein wesentliches Gewicht. Schon in Kants erster Kritik wird der transzendentalen Apperzeption des Ich (und der dadurch begründeten synthetischen Urteile apriori) eine ursprüngliche Synthesis der Einbildungskraft als Bedingung zugrunde gelegt. ${ }^{42}$ Dem Vollzug dieser unhintergehbaren Syntheseleistung ist die Gegebenheit von Vorstellungen überhaupt erst zu verdanken - auch derjenigen Vorstellungen, die das Ich von sich selbst hat. Einen Bezug zu sich selbst kann das Subjekt mithin schon auf der Ebene des Gefühls allein über den Umweg der Einbildung herstellen.

Im ersten Paragraphen der dritten Kritik wird erneut die Einbildungskraft als die unhintergehbare Voraussetzung eines jeden Urteilsvollzugs und Selbstbezugs hervorgehoben. Das "Subjekt» und "das Lebensgefühl desselben«, die die Urteilskraft begründen, sind darauf angewiesen, »durch die Vorstellung affiziert" zu werden, die ihnen "durch die Einbildungskraft" gegeben wird. ${ }^{43}$ Dass es Bilder der Einbildungskraft sind, die den Fremd- und Selbstbezug des Subjekts konstituieren, wird später noch einmal mit aller Nachdrücklichkeit behauptet. Der »intellectus ectypus « des Menschen ist - im Gegensatz zum »intellectus archetypus«

4I Immanuel Kant: Kritik der Urteilskraft. Hg. von Wilhelm Weischedel. Frankfurt a. M. 1974, \$9.

42 Kant: Kritik der reinen Vernunft (s. Anm. 36), B 104.

43 Kant: Kritik der Urteilskraft (s. Anm. 4I), \$ I. 
Gottes - auf »Bilder « angewiesen. ${ }^{44}$ Sie bieten die Anlässe, die dem menschlichen Denken die Selbstaffizierung erlauben, die Kant in seiner Untersuchung der ästhetischen Urteilskraft als notwendige Bedingung eines jeden Urteilsvollzugs nachzuweisen sucht. Daher auch Kants Hinwendung zum ästhetischen Urteil. Wird geurteilt, diese Rose sei schön, so ist damit (wie man weiß) nichts über die Rose selbst gesagt, sondern nur über das Selbstgefühl des urteilenden Subjekts. Folglich kommt in einem solchen Urteilsvollzug der Anlass, der Vorstellungen gibt (und damit überhaupt erst objektive Aussagen über Gegenstände ermöglicht), eigens in den Blick. Der Fremdbezug, der jeden Selbstbezug grundiert, wird bei Kant indes ständig marginalisiert. Er darf mithin nur die Veranlassung für das Urteil abgeben, nicht aber in das Urteil mit eingehen. Daher kann Walter Benjamin unterstreichen, dass in »Kants Theorie vom freien Spiel der Gemütskräfte der Gegenstand als ein Nichts zurücktritt, um nur den Anlaß einer selbsttätigen, inneren Stimmung des Geistes zu bilden «. ${ }^{45}$

Noch Fichte wird die Notwendigkeit eines solchen Katalysators zugestehen, der das Subjekt zu sich selbst bringt, aber nicht selbst in das Subjekt eingeht. Schließlich wäre seine unendliche Aufgabe keine »aufgegebne ${ }^{46}$, würde sie nicht durch einen »Anstoß « angetrieben. ${ }^{47}$ In Fichtes kausaler Argumentation bleibt die Rolle dieses Anstoßes auf die einer causa efficiens beschränkt, die nicht in die Zielsetzung (die causa finalis) einwandern darf. Weil sonst keine Freiheit des Ichs denkbar wäre, erschöpft sich der Anstoß in der bloßen Faktizität, »daß überhaupt ein System sein solle «. ${ }^{4}$ Bei Kant dagegen ist die Rolle des Anlasses noch weit weniger klar umrissen, damit aber auch weit gewichtiger. Selbst die alles entscheidenden synthetischen Urteile apriori müssen von ihnen äußerlich bleibenden Anlässen initiiert werden. Dem verleiht Kant dadurch Ausdruck, dass er diesen Urteilen neben einer begrifflichen noch eine gleichsam materiale Komponente

44 Ebd., $\$ 77$.

45 Benjamin: Der Begriff der Kunstkritik (s. Anm. 8), S. 63 f.

46 Fichte: Grundlage der gesamten Wissenschaftslehre (s. Anm. 39), S. 207.

47 Ebd., S. I29.

48 Ebd., S. 36. 
beifügt. ${ }^{49}$ Eben dieser Angewiesenheit auf ein Außen wegen kann es bei Kant auch kein Fichte'sches absolutes Ich geben. Seiner selbst gewahr wird das Ich bei Kant immer nur durch die Vermittlung der Einbildungskraft.

Schlegel nun wird den Anlasscharakter von Denken und Urteilen in einer Konzeption von Cyklisierung stark machen, die ihre stranszendentale Materialität mit in das Programm aufnimmt und damit auch den Fremdbezug, der jeden Selbstbezug ermöglicht (oder, mit Hamacher gesprochen: die »Heteroaffektion «, die jede

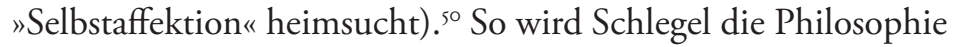

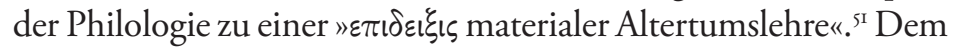
"Herabsteigen" des Fichte'schen Idealismus will er damit eine "Totalisazion von unten herauf « entgegensetzen können..$^{52}$ In der solchermaßen vom Kopf auf die Füße gestellten "Cyklisazion «s3 werden zwei Ergebnisse der Fichte-Lektüre produktiv gemacht: Erstens die Einsicht, dass Fichtes Kreisgang notwendig eine Vielzahl von "Lücken «" lässt und dass sein Begriff der Perfektibilität demnach "nicht bloß extensiv sondern auch intensiv «s gefasst werden müsste; sowie zweitens die Überzeugung, dass die Gefahr, die der Annahme eines absoluten Ichs ständig drohe, "ein dicker empirischer Egoismus der theoretischen Art ${ }^{56}$ sei, welcher nicht nur die Eigenwürde des Nicht-Ichs, sondern auch die eines jeden anderen empirischen Ichs in Abrede stelle.

Um mit dem philosophischen Modell des Kreisgangs zu brechen, bringt Schlegel nun die Rhetorik des genos epideiktikon ins Spiel.

49 Auf diese transzendentale Materialität hat zuerst Martin Heidegger im Zusammenhang einer Analyse der Selbstaffektion in Kants Kritik der reinen Vernunft hingewiesen; siehe Martin Heidegger: Kant und das Problem der Metaphysik. 4. Auf. Frankfurt a. M. 1991, S. 138-203. Aufgegriffen wurde die Rede von der transzendentalen Materialität in Paul de Man: „Kants Materialism«. In: ders.: Aesthetic Ideology (s. Anm. 4), S. II9-I28. Hamacher: »Für - die Philologie« (s. Anm. I), S. 4I und 34.

5I Schlegel: Zur Philologie I (s. Anm. Io), S. 48, Nr. 156.

52 Schlegel: Zur Philologie II (s. Anm. 30), S. 68, Nr. 84.

53 Ebd.

54 Friedrich Schlegel: Philosophische Fragmente I796. In: KFSA I8, S. 505-516, hier S. 509, Nr. 45.

55 Ebd., S. 507, Nr. 15.

56 Ebd., Nr. 3I. 

Anderen nur als äußeren Anstoß zuließ, in das weit komplexere Wechselverhältnis von res und verba überführt, das die Rhetorik grundlegend bestimmt. Dank der Übertragung in ein rhetorisches Register kann der Anstoß zum Anlass aufgewertet werden, ohne dass eine durch ihn initiierte philosophisch-philologische Forschung von ihm wie von einer Ursache bewirkt würde. Diese Spannung von Freiheit und Bedingtheit ist es, was Schlegel mit der epideixis gewinnt. Damit kann einem nahe liegenden Vorurteil begegnet werden, das in einer solchen Bezugnahme auf die Rhetorik Alteuropas nichts als eine Rückwendung zum ancien régime sehen würde. In dieser Weise ist Schlegels Geste aber nicht zu deuten, vielmehr geht es ihm darum, die spannungsvolle Anlasshaftigkeit philologischer Forschung eigens ins Auge zu fassen.

Vor dem Hintergrund seiner Kritik am deutschen Idealismus kommt zugleich der spezifische `Materialismus` von Schlegels materialer Altertumslehre in den Blick: es geht, wie sich nun zeigen wird, um eine unbegrenzte Ausweitung sowohl des Gegenstandsbereichs der Philologie als auch derjenigen, die sie betreiben. Die Anlassgebundenheit philologischer Forschung hat also ganz konkrete Folgen. Schlegel zufolge macht nämlich Philologie eine potenziell selbst anlassgebundene Dichtung zum Anlass einer philologischen Operation oder Intervention - und bietet somit wiederum den Anlass für weitere philologische Operationen und Interventionen. Auf diese Weise werden nicht nur immer neue Arbeiten angestoßen, vielmehr wird auch die Struktur des Zirkels im Beweis bei Schlegel verändert. Durch jede neue Forschungsarbeit, die den Kreis der Schlegel'schen Philologie zu schließen versucht, indem sie an ihn anschließt, wird er zugleich immer auch unterbrochen. Wohl auch mit Blick auf diese Struktur philologischer Arbeit wird Walter Benjamin in seiner Studie über die Kunstkritik der Romantik vom "paradoxen Versuch « sprechen, "am Gebilde noch durch Abbruch zu bauen «. ${ }^{57}$ Eine solche Arbeit ist allein der "Unendlichkeit des Zusammenhangs « angemessen, die

57 Benjamin: Der Begriff der Kunstkritik (s. Anm. 8), S. 87. 
Benjamin eingangs von der »Unendlichkeit des Fortgangs «abheb $\mathrm{t}^{{ }^{8}}$ und damit die Unterscheidung von sintensiver Perfektibilität in ein Forschungsprogramm übersetzt.

Wenn Schlegel die enzyklopädische Perspektive seines philologischen Projekts als unendliche Aufgabe ansieht, dann beruht deren Unabschließbarkeit demnach nicht wie bei Fichte darin, dass die Unendlichkeit nur näherungsweise erreichbar ist, sondern darin, dass der Kreis in eine unendliche Zahl von Kreisen zerfällt. Und das gilt sowohl für die Seite des Gegenstands wie die des Trägers philologischer Forschung. Schlegel bezieht somit gewissermaßen die Gegenposition zu Hegel, der den Kreisgang Fichtes in einem enzyklopädischen "Kreis von Kreisen «" zu schließen suchen wird. Bei Schlegel müsste man im Gegenteil davon sprechen, dass diese Kette in eine unendliche Vielzahl von Kreisen zerspringt, von denen ein jeder den Anlass für die Bildung immer neuer, sich dezentral streuender Kreise bieten kann. Das enzyklopädische Projekt des I8. Jahrhunderts fällt so einem philologischen Okkasionalismus anheim, der sich für alles und jeden öffnen will. Dieser Okkasionalismus ist doppelt gelagert: Der Gegenstandsbereich wird auf eine transdisziplinäre kulturwissenschaftliche Perspektive hin geöffnet. Dieser Öffnung entspricht eine unbestimmte Ausweitung der am philologischen Projekt Beteiligten. Weil diese beiden Ausweitungen im Denken Schlegels über die Figur des gebrochenen Kreises der Enzyklopädie miteinander verschränkt sind, zeichnet sich eine für ihn offenbar notwendige Ko-Implikation von Kulturwissenschaft und Kulturpolitik ab, die eine gesellschaftlich wie kulturell emanzipatorische Stoßkraft entfalten soll.

58 Ebd., S. 26. In diesem Sinne lässt auch Werner Hamacher Schlegel »alle Zyklen zersprengen " und damit "nicht weniger als die Totalität sämtlicher sprachlicher Setzungen suspendieren «; auf solche Weise richtet die Schlegel'sche Philologie »ihre Kritik endlich gegen die Borniertheit der Totalität dessen, was in den überschaubaren historischen Gebieten überhaupt als Sprache und als Literatur gilt» (Hamacher: »Für- die Philologie« [s. Anm. I], S. 37).

59 Georg Wilhelm Friedrich Hegel: Wissenschaft der Logik. Hg. von Eva Moldenhauer u. Karl Markus Michel. Frankfurt a. M. 1986, S. 57I. 
3. Eine okkasionalistische Enzyklopädie

Schlegels Kritik an der Philologie seiner Zeit ist analog zu derjenigen an der Philosophie. Auch jene schotte sich gegen den Anlass ab, den sie gleichwohl in Anspruch nehmen müsse: Weil die klassischen Werke für die Philologen eine bloß "äußre Veranlassung" sind, ${ }^{60}$ kennen sie "das Alterthum (der Materie nach) « sogar am wenigsten von allen. ${ }^{6}$ Ausschlaggebend ist also auch hier, dass der Anlasscharakter an den Rand gedrängt wird. Denn damit ändert sich der epideiktische Charakter der Philologie, die nicht mehr (wie die an der Rhetorik geschulte Literatur) und noch nicht (wie die von Schlegel für die Zukunft erwartete Philosophie der Philologie) ein »Publ[icum] « hat und sich als »Fest « inszeniert. ${ }^{62}$ Die Epideiktik wandert in kleine Kreise, die ihre akademischautonomen Feste feiern. Dagegen hebt Schlegels Popularisierung der Philologie gerade auf einen öffentlichen Festcharakter ab, weil er sich davon die Öffnung des gelehrten und universitären Betriebs für alle gesellschaftlichen Schichten und alle Geschlechter verspricht. Anders gesagt muss die Philologie, um wieder eine gesamtgesellschaftliche Rolle spielen zu können, den Anlass ihrer epideixis nicht nur als äußeren 'Anstoß verstehen, sondern auch als innere Veranlassung - mithin als ihren 'Zweckı. Dies gelingt gerade einer zyklisch-enzyklopädischen Methode, deren offenen, sich streuenden Kreisen eine unlösbare Spannung von »Veranlassung« und "Zweck« (die »beydes nur Eins« sein sollen) innewohnt. ${ }^{63}$

Obwohl sie sich mit der epideixis bei einer poetisch-rhetorischen Figuration der höfischen Gesellschaft bedient, verschreibt sich

60 Schlegel: Zur Philologie I (s. Anm. Io), S. 53, Nr. 207.

6I Ebd., S. 42, Nr. 9I.

62 Ebd., S. 46, Nr. I4I. Bei Schlegel: Über das Studium der Griechischen Poesie (s. Anm. I6), S. 277, heißt es: "Dichtung, Gesang, Tanz und Geselligkeit festliche Freude war das holde Band der Gemeinschaft, welches Menschen und Götter verknüpfte. Und in der Tat war auch der Sinn ihrer Sage, Gebräuche und besonders ihrer Feste, der Gegenstand ihrer Verehrung das echte Göttliche: die reinste Menschheit."

63 Schlegel: Zur Philologie I (s. Anm. Io), S. 53, Nr. 206. 
Schlegels Popularisierung der Philologie keiner restaurativen Tendenz - jedenfalls um I80o noch nicht. Auch nicht in dem Sinn, dass sie die verlorene Einheit der alteuropäischen Gesellschaft restituieren will. Schlegel entspricht der funktionellen Ausdifferenzierung der modernen - posthöfischen - Gesellschaft durch die Ansetzung eines $" \phi \lambda\left[\right.$ philologisch] Absolute $[n] \ll^{64}$, die die mikrologische Arbeit am Material gegen die Ästhetik eines abstrakt-absoluten Transzendentalismus ${ }^{65}$ mobilisiert. Bereits das kleinste $» \phi \mu[$ Philologem] « sei enzyklopädisch, weil es »sich auf eine unermeßliche Menge von bedingt[en], ja oft höchst mikrologischen Kenntnissen " bezieht ${ }^{66}$ und so "nach unendlich vielen Seiten in unendlich vielen Richtungen auf das philologisch Absolute bezogen werden « kann. ${ }^{67}$

Diese Mikrologie philologischer Forschung wird zugleich Benjamins »Unendlichkeit des Zusammenhangs ${ }^{68}$ gerecht und Hamachers »immanente[r] Suspendierung einer jeweils erreichbaren Totalität «. ${ }^{69}$ Sie hat eine radikale Transdisziplinarität zur Folge, die verdeutlicht, warum sich die Philologie bei Schlegel als materiale Altertumslehre begreift. Philologische Forschung verfügt nicht über ein bestimmtes Set von Methoden, sondern passt ihre Verfahrensformen dem jeweiligen Anlass an. Die methodische bricolage geht so weit, dass Schlegel betont: die »Gesetze der $\phi \lambda[$ Philologie] sind sämmtl[ich] entlehnt «.70

Die Auswirkungen, die Schlegels Konzeption auf die Philologie des frühen 19. Jahrhunderts hatte, lassen sich etwa am Philologiebegriff August Boeckhs ermessen. Boeckh, ein Schüler Wolfs und Schleiermachers, hat das Schlegel'sche Projekt, wenngleich innerhalb gewisser Grenzen, umgesetzt. ${ }^{\text {I }}$ Sein Versuch,

64 Schlegel: Zur Philologie II (s. Anm. 30), S. 67, Nr. 77.

65 Siehe dazu Schlegel: Zur Philologie I (s. Anm. Io), S. 37, Nr. 33.

66 Schlegel: Zur Philologie II (s. Anm. 30), S. 66, Nr. 77.

67 Ebd., Nr. 79.

68 Benjamin: Der Begriff der Kunstkritik (s. Anm. 8), S. 26.

69 Hamacher: "Für- die Philologie« (s. Anm. I), S. 38.

70 Schlegel: Zur Philologie I (s. Anm. Io), S. 48. Nr. 158.

7I Zur Beziehung von Schlegel und Boeckh siehe Nikolaus Wegmann: „Was heißt einen ‘klassischen Text lesen? Philologische Selbstreflexion zwischen Wissenschaft und Bildung«. In: Jürgen Fohrmann/Wilhelm Voßkamp (Hg.): 
die Transdisziplinarität der Philologie zu systematisieren, findet sich in der grundlegenden Enzyklopädie und Methodenlehre der Philologischen Wissenschaften, deren "materiale Altertumskunde" die für die Philologie relevantesten Wissensgebiete abhandelt. ${ }^{72}$ Boeckhs Ansatz einer historisch-kulturwissenschaftlich ausgebauten Philologie übernimmt aber nicht nur den mikrologischen Ansatz philologischer Arbeit, sondern tritt auch deren rhetorisches Erbe an. Wenn er die historische gegen die mathematische Wahrheit abhebt (also das, was man später geisteswissenschaftliche Wahrheit nennen wird, gegen die naturwissenschaftliche), tut er dies im Rückgriff auf die Argumentationsweise, die Aristoteles der Rhetorik (im Gegensatz zur Wissenschaft) vorbehalten hatte: den enthymemischen Schluss aus Prämissen der Wahrscheinlichkeit, Annehmlichkeit und Glaubwürdigkeit. ${ }^{73}$

Nun zeichnet sich aber ein doppelter Unterschied von Boeckhs Enzyklopädistik zu Schlegels `Cyklisazion`ab. Zum einen blendet Boeckh die rhetorischen Grundlagen seiner Philologie aus und betont stattdessen - wie vor ihm schon Schleiermacher - die divinatorisch-genialische Komponente historischer Erkenntnis ${ }^{74}$; zum anderen versucht er der sich ins Unermessliche streuenden methodischen Pluralität der Philologie dadurch Herr zu werden, dass er sie disziplinär bündelt. Dagegen streicht der Schlegel der Hefte Zur Philologie die epideiktisch-rhetorische Verhaftung philologischer Argumentation heraus, offenbar weil für ihn die Chancen gerade in der Dezentralisierung der Kontexte und nicht in Versuchen ihrer neuerlichen Einhegung liegen.

Wissenschaftsgeschichte der Germanistik im I9. Jahrhundert. Stuttgart/Weimar 1994, S. 334-450, hier S. 371-398, sowie jetzt Mirco Limpinsel: „Diaskeuasen des Geistes. Perspektiven auf den philologischen Gegenstand bei Friedrich Schlegel, Wolf, Ast und Boeckh». In: Breuer/Bunia/Erlinghagen (Hg.): Friedrich Schlegel und die Philologie (s. Anm. 5), S. I45-164.

72 Siehe hierzu August Boeckh: Enzyklopädie und Methodenlehre der Philologischen Wissenschaften. Hg. von Ernst Bratuscheck. Bd. 2. Stuttgart I960.

73 Ebd., $\$ 30$. Für diese rhetorische Argumentationsform siehe Aristoteles: Ars rhetorica (s. Anm. I2), I356b-1357b.

74 Boeckh: Enzyklopädie und Methodenlehre der Philologischen Wissenschaften (s. Anm. 72), \$32. 
Diese beiden Aspekte hängen bei Schlegel zuinnerst zusammen. Seinem radikal transdisziplinären kulturwissenschaftlichen Ansatz hofft er offenbar deshalb mit einer philologischen epideixis gerecht werden zu können, weil diese in Analogie zur antiken Anlassdichtung konzipiert ist. Wo Pindar den konkreten Glücksfall eines Sieges in eine größere Geschichte einzubetten suchte, indem er unter einer Reihe von Mythen und Mythenvarianten auswählte (und dabei im Übrigen oft ganz ausdrücklich die eine auswählt und andere ausscheidet), ${ }^{75} \mathrm{da}$ wählen Schlegels Philologinnen und Philologen aus dem ganzen Fächer von Begriffen und Narrativen, Techniken und Analysemethoden aus, die ihnen zur Verfügung stehen. Nur so können sie die "Außerordentl. [iche] Mannichfaltigkeit an Gelegenheit zur Philologie « ${ }^{76}$ bewältigen, die Schlegel konstatiert. Philologie wird also Gelegenheitsphilologie.

Der Ausdruck >Mannichfaltigkeit`verweist erneut auf die Philosophie Kants. In der Kritik der reinen Vernunft wird mit Mannigfaltigkeit der Zustand der (Außen- und Innen-)Welt vor der basalen Syntheseleistung der Einbildungskraft bezeichnet. ${ }^{77}$ Wenn der Schlegel'sche Text das Wort `Mannigfaltigkeit im Zusammenhang mit der 'Gelegenheit zur Philologie` fallen lässt, wird damit der "Anlas[s] « der "gegebenen Vorstellung « ${ }^{78}$ aus der Kritik der Urteilskraft evoziert, von dem oben bereits gezeigt wurde, dass er in der Veranlassung zur Syntheseleistung der Einbildungskraft liegt. Eine ähnliche Rolle spielt der Begriff der Mannigfaltigkeit in Schlegels wohl wenig früher entstandener Schrift Über das Studium der Griechischen Poesie. Dort wird die Überzeugung, dass die "Mannigfaltigkeit« schon vor jeder Subsumtion unter Gesetzlichkeiten »die erste Form « und »nicht roher Stoff « sei, ${ }^{79}$ zur Legitimation dafür, dass man die Griechen "in Masse studiren" solle. ${ }^{80}$ Christian Benne hat auf die zentrale Bedeutung dieser

75 Grundsätzliches hierzu findet sich etwa bei John T. Hamilton: Soliciting Darkness. Pindar, Obscurity, and the Classical Tradition. Cambridge 2003, S. $77-96$.

76 Schlegel: Zur Philologie I (s. Anm. IO), S. 4I, Nr. 83.

77 Kant: Kritik der reinen Vernunft (s. Anm. 36), B I04.

78 Kant: Kritik der Urteilskraft (s. Anm. 4I), \$9.

79 Schlegel: Über das Studium der Griechischen Poesie (s. Anm. I6), S. 290.

80 Ebd., S. 347. 
Wendung - auch über den Studium-Essay hinaus - aufmerksam gemacht und sie in Zusammenhang mit dem Begriff der Organisation erörtert, wie er im zweiten Teil der Kritik der Urteilskraft entwickelt wird. ${ }^{81}$ Kant benutzt dort das Wort Organisation, um die von der teleologischen Urteilskraft unterstellte "innere Naturvollkommenheit« der "Naturzwecke« zu charakterisieren, in denen alles zugleich Mittel und Zweck sei. ${ }^{82}$

Was Bennes Aufsatz unberücksichtigt lässt, ist die politische Bedeutung, die sowohl im Masse- als auch im Organisationsbegriff ${ }^{3}$ mitschwingt. Spätestens seit Gentz' Übersetzung von Edmund Burkes Reflections on the Revolution in France, die das englische "crowd" als "Masse" wiedergibt, ${ }^{84}$ hat das Wort eine politische Konnotation. Ebenso verhält es sich mit `Organisation`, wie einer Fußnote zu entnehmen ist, die just dem zuletzt zitierten Paragraphen der Kritik der Urteilskraft beigefügt ist. Darin bemerkt Kant mit Blick auf die Amerikanische Revolution, man habe sich

bei einer neuerlich unternommenen gänzlichen Umbildung eines großen Volkes, zu einem Staat, des Wortes Organisation häufig für die Einrichtung der Magistraturen u.s.w. und selbst des ganzen Staatskörpers sehr schicklich bedient. ${ }^{85}$

8I Siehe Christian Benne: „Kunst der Organisation. Zur Philologie der `Massen in Friedrich Schlegels Über Goethes Meister«. In: Breuer/Bunia/Erlinghagen (Hg.): Friedrich Schlegel und die Philologie (s. Anm. 5), S. I09-II2.

82 Kant: Kritik der Urteilskraft (s. Anm. 4I), \$65.

83 Dass die politische Dimension des Organisationsbegriffs, wie er sich um I80o herausbildet, wichtiger ist als die organizistisch-natürliche Dimension wird schon betont in Niklas Luhmann: Organisation und Entscheidung. Opladen 1978 , S. 5 .

84 Siehe hierzu Susanne Lüdemann: „Zusammenhanglose Bevölkerungshaufen, aller inneren Gliederung bar`. Die Masse als das Andere der Ordnung im Diskurs der Soziologie«. In: Behemoth. A Journal on Civilisation 7.I (20I4), S. I03-II7, hier S. IO4.

85 Kant: Kritik der Urteilskraft (s. Anm. 4I), \$65. Ausgehend von dieser Stelle entwickelt Hannah Arendt das (in Amerika versprochene, aber nicht umgesetzte) Modell einer post-souveränen Ordnung. Siehe Hannah Arendt: Lectures on Kant's Political Philosophy. Hg. von Ronald Beiner. Chicago I992, S. I6. 
142 Eine vergleichbare politische Dimension ist auch in Schlegels Gebrauch des Wortes 'Masser nachweisbar. Denn die Griechen "in Masse studiren « heißt nicht nur, sich von der Fixierung auf die hervorragenden Vertreter griechischer Literatur loszusagen, weil »die ganze Griechische Bildung überhaupt [...] ein solches Ganzes [ist], welches nur in Masse erkannt und gewürdigt werden kann. ${ }^{86} \mathrm{Um}$ einer solchen Forderung nachkommen zu können, ist auch gleichsam eine levée en masse von Studierenden nötig, die die anfallende (in den Worten der Fichte-Studien: sintensiv-absolutes) Forschungslast schultern könnte. Denn es geht schließlich darum, dass eine neue "ästhetische Bildung " ausgehend von » recht vielen einzelnen Punkten« sich »über die ganze Masse« der Gesellschaft erstreckt. ${ }^{87}$ Nur dadurch könne die bisherige "politisch[e] Pfuscherei« beendet werden. ${ }^{88}$

Beide Bedeutungen verschränken sich in Schlegels Gebrauch der Wendung, sodass sich hier der innere Nexus abzeichnet, der die kulturwissenschaftliche Fundierung und die kulturpolitische Zielsetzung der Philologie verknüpft. Allerdings wird der MasseBegriff in den Heften Zur Philologie keine Rolle mehr spielen. Das liegt aber keineswegs daran, dass dieser Text die Verbindung von Kulturwissenschaft und Kulturpolitik aufkündigt, sondern daran, dass er den emanzipatorischen Zug einer sich selbst organisierenden Philologie noch expliziter herausarbeitet.

4. Von Kants sensus communis aestheticus zu Schlegels sensus communis philologicus

Die Genese der Selbst-Organisation von PhilologInnen ist durch die "Mannichfaltigkeit an Gelegenheit zur Philologie " $^{89}$ in einer solchen Weise veranlasst, dass die Mannigfaltigkeit des Anlasses auch eine Mannigfaltigkeit der Forschenden fordert. Nun ist abschließend zu untersuchen, wie diese Mannigfaltigkeit zu

86 Schlegel: Über das Studium der Griechischen Poesie (s. Anm. I6), S. 347.

87 Ebd., S. 360.

88 Ebd.

89 Schlegel: Zur Philologie I (s. Anm. Io), S. 4I, Nr. 83. 
denken ist und inwiefern sie sich von einer schlichten $>$ Masse von Forschenden unterscheidet. Damit kommt zugleich die Art von Popularisierung in den Blick, in deren Dienst sich die Hefte Zur Philologie stellen. Hier kann man wiederum von Schlegels Aufwertung des Anlasses im Vergleich zu Kant ausgehen.

Wie oben auseinandergesetzt, sagt ein Geschmacksurteil bei Kant nichts über seinen Gegenstand, sondern etwas über die durch den Gegenstand veranlasste (Selbst-)Affizierung des urteilenden Subjekts aus. Weil `Subjekt` hier (wie auch sonst bei Kant) die Struktur des menschlichen Daseins überhaupt meint, kommt solchem ssubjektiven`Urteile gleichwohl Allgemeingültigkeit zu. Wenn und sofern man ein solches Urteil ausspricht, erhebt man "Anspruch « auf "eine allgemeine Stimme«..$^{\circ \circ} \mathrm{Die}$ Allgemeingültigkeit der Stimme erschöpft sich mithin im Akt des Urteilens, der sich schlicht dadurch legitimieren will, dass er vollzogen wird. Der Zwiespältigkeit dieser petitio principii geschuldet ist nicht nur Kants scheinbar widersprüchliche Rede davon, dass man den anderen sein Urteil "ansinnt " ${ }^{91}$ zugleich aber um ihre Zustimmung "wirbt «.92 Darüber hinaus erklärt diese Zwiespältigkeit den prekären, weil zugleich notwendigen und unmöglichen, Status dessen, was er als sensus communis aestheticus bezeichnet. Zwar wird ein solcher Gemeinsinn jedes Mal, wenn man ein Geschmacksurteil fällt, notwendig in allen empirischen Ichs vorausgesetzt, doch kann er nie empirische Wirklichkeit gewinnen. Weil er trotzdem ein Sinn sein soll, ${ }^{93}$ gehen im sensus communis Transzendentales und Empirisches auf eine nicht zu klärende Weise ineinander über. ${ }^{94}$

Anders als der deutsche Idealismus, den dieses Dilemma schließlich zur Formulierung einer geschichtsphilosophischen Dialektik von empirischem und transzendentalem Ich nötigt, macht Schlegel die paradoxe Struktur des Gemeinsinns dadurch

90 Kant: Kritik der Urteilskraft (s. Anm. 4I), \$8.

9I Ebd., \$3I.

92 Ebd., \$ I9.

93 Siehe ebd., \$2I.

94 Bekanntlich soll die Urteilskraft den »Übergang« von dem Verstand, der "Erscheinungen« bestimmt, zu der Vernunft, die es mit dem »Ding an sich" zu tun hat, leisten (ebd., Einleitung, II). 
144 fruchtbar, dass er die Akzentsetzung von der universalen Stimme Kants auf die Mannigfaltigkeit der Anlässe verschiebt. Weil das in den Heften Zur Philologie skizzierte philologisch-philosophische Projekt damit die (gleichermaßen empirische wie transzendentale) Materialität ihrer Anlässe mitreflektiert, historisiert sie nicht nur den Kantischen Begriff der Transzendentalität, sondern auch ihren Vollzug der Urteilskraft. ${ }^{95}$ Um dies am Beispiel der Konjekturalkritik zu konkretisieren: die Notwendigkeit der Konjektur wird durch den historischen Abstand zwischen Text und Leser und die fehlerhafte historische Überlieferung »veranlaßst.. ${ }^{96}$ Diesem Anlass entspringt die Antinomie von Kritik und Hermeneutik, deren Widerstreit von Rezeptivität und Spontaneität, von Hineinlesen und Auslegen, nur im Vollzug des Urteils (zwar nicht aufgehoben, aber) produktiv gemacht werden kann. In einem solchen Urteilsvollzug bekundet sich ein philologischer sensus communis, der im Gegensatz zu seiner Kantischen Spielart um seine historische Verortung und seinen kulturellen bias weiß.

Das philologische Urteil setzt eine Kulturpolitik in Gang, die durch die kulturwissenschaftliche Ausweitung der 'Gelegenheit zur Philologie enzyklopädisch nun auch in dem Sinne wird, dass sie sich allen zuwendet und für alle öffnet. Diese Popularisierung beruht indessen nicht darauf, dass einfach immer größere Massen von Philologen in einen Kreisgang eingeschlossen würden. Die Struktur der Popularisierung ist vielmehr so beschaffen, dass ein jeder den Kreis öffnet, der ihn zu schließen sucht, indem er an ihn anschließt. Werner Hamacher wird diesen paradoxen (weil nicht-institutionalisierten) Sprechakt der Philologie als ein Sprechen charakterisieren, »das aber selber nichts sagt außer dem Anfang des Sagens. ${ }^{97}$ Darin bekundet sich der Entwurf einer nicht-institutionalisierbaren Philologie, die nicht nur - und nicht vorrangig - eine universale politische Stimme für sich be-

95 Nicht zuletzt deshalb wird Foucault Schlegel als Gewährsmann für die sprachliche Dimension der empirisch-transzendentalen Doublette anführen. Siehe Michel Foucault: Die Ordnung der Dinge. Eine Archäologie der Humanwissenschaften. Übers. von Ulrich Köppen. Frankfurt a. M. I974,

S. 342 und 384 .

96 Schlegel: Zur Philologie II (s. Anm. 30), S. 78, Nr. 192.

97 Hamacher: "Für - die Philologie» (s. Anm. I), S. 39. 

emanzipatorische Rolle spielen will, indem sie eine dezentrale Organisation hervorbringt, wie sie in der dritten Kritik weniger im Ausgang von natürlichen Phänomenen als von politischen Umwälzungen entwickelt wird.

Solche Überlegungen führen unweigerlich zu Fragen der ‘politischen Romantikı. Für Carl Schmitt, der diesen einflussreichen Begriff in einem gleichnamigen Buch geprägt hat, würde der so auf einem Okkasionalismus fußende politisch emanzipatorische Charakter der Philologie dem Bann verfallen. Denn er wäre ein weiteres Beispiel für die von Schmitt heftig kritisierten allgemein romantischen Bestrebungen, "die Hierarchie der geistigen Sphäre» aufzulösen und potenziell "alles zum Zentrum des geistigen Lebens werden« zu lassen. ${ }^{98}$ Als Motivation dieser dezentralisierenden Einebnung geistiger Hierarchien macht er eine Usurpation des "romantischen Subjekts" aus, das keine anderweitige Souveränität zugibt, weil es »statt Gottes« die »zentrale Stelle» im Weltganzen

einnimmt und aus der Welt und allem was in ihr geschieht, einen bloßen Anlaß macht. Dadurch, daß die letzte Instanz sich von Gott weg in das geniale "Ich" verlegt, ändert sich der ganze Vordergrund und tritt das eigentlich Occasionalistische rein zutage. [...] Aus immer neuen Gelegenheiten entsteht eine immer neue, aber immer nur occasionelle Welt, eine Welt ohne Substanz und ohne funktionale Bindung, ohne feste Führung, ohne Konklusion und ohne Definition, ohne Entscheidung, ohne letztes Gericht [...].99

Obwohl Schmitt hier die Folgen einer politischen Romantik, wie sie unter anderem von Schlegels philologischem Projekt forciert wird, mit allem Nachdruck darstellt, entgeht ihm ihre eigentlich revolutionäre Stoßkraft. Es geht nicht darum, die Systemstelle Gottes oder des Souveräns zu besetzen, sondern sie, wenn sie schon nicht einfach abgeschafft werden kann, frei zu halten - auch und gerade von den Anmaßungen des (sei es nun empirischen

98 Carl Schmitt: Politische Romantik. 6. Aufl. Berlin I998, S. I7.

99 Ebd. 
146 oder transzendentalen) Ichs. ${ }^{100}$ Ja mehr noch: diese Systemstelle wird nicht nur freigehalten, sondern ganz bewusst - als eine Leerstelle - zerstreut.

\section{Schlegel - für die Philologie}

Schlegels Entwurf einer sich selbst dezentral organisierenden Philologie steht nicht umsonst Pate für Werner Hamachers Intervention Für - die Philologie: nimmt er doch an, philologische Arbeit sei schon in sich eine politisch subversive Praxis. Das gilt, wie eingangs formuliert, auch für Paul de Man. ${ }^{101}$ Abschließend sollen einige der

Ioo Dafür, dass sich die moderne Demokratie dadurch auszeichnet, dass sie den Ort der Macht freihält, argumentiert bereits Claude Lefort: "L'image du corps et le totalitarisme«. In: ders.: L'invention démocratique. Les limites de la domination totalitaire. Paris 1981, S. I59-176. Unter anderem auch deswegen waren die Romantiker von der Sedisvakanz des Heiligen Stuhls zwischen dem Pontifikat von Pius VI. und Pius VII., die I799/180o ungewöhnlich lange dauerte, so angezogen. Im Hinblick auf Novalis wurde die Bedeutung dieser Sedisvakanz schon von Ludwig Stockinger betont, der hier eine Anregung von Hans-Joachim Mähl aufnimmt. Siehe etwa Ludwig Stockinger: „Religiöse Erfahrung zwischen christlicher Tradition und romantischer Dichtung bei Friedrich von Hardenberg (Novalis) «. In: Walter Haug/Dietmar Mieth (Hg.): Religiöse Erfahrung. Historische Modelle in christlicher Tradition. München 1992, S. 36I-393, hier S. 384, sowie Hans-Joachim Mähl: „Kommentar zu `Die Christenheit oder Europa«". In: Novalis: Werke, Tagebücher und Briefe Friedrich von Hardenbergs. Hg. von dems. u. Richard Samuel. Bd. 3. München 1987, S. 579-604, hier S. 603. Wie Ulrich Breuer in einem Aufsatz nahelegt, spielte Napoleons Staatsstreich, durch den das Directoire abgesetzt wurde und Frankreich eine Nacht lang keine Führung hatte, für das berühmte Jenaer Romantikertreffen eine gewichtige Rolle. Siehe hierzu Ulrich Breuer: "Making-of. Zur Vorgeschichte von Friedrich Schlegels Gespräch über die Poesie«. In: Dirk von Petersdorff/Ulrich Breuer (Hg.): Das Jenaer Romantikertreffen im November I799. Ein romantischer Streitfall. Paderborn u. a. 2015 (=Athenäum. Sonderheft), S. I39-I66. Im selben Band findet sich ein Beitrag von Ludwig Stockinger, in dem es unter anderem um die Bedeutung der Sedisvakanz für das Romantikertreffen geht.

IOI Für eine eingehendere Darstellung der Bedeutung, die die epideiktische Anlassdichtung für Paul de Man hat, siehe Michael Auer: "Anlass und Deutung. Gelegenheitsdichtung bei Paul de Man«. In: Thomas Vesting/ Susanne Lüdemann (Hg.): Was heißst Deutung? München 2016 [i. Dr.]. 
Folgen entwickelt werden, die der explizite Rückgriff auf Schlegel und der darin implizierte Rekurs auf einen epideiktischen (und keinen hermeneutischen) Zirkel beinhaltet.

Die Form von Aufklärung und Demokratisierung, die Philologie offenbar für de Man und Hamacher (wie zuvor für Schlegel) verspricht, ist weder einem Repräsentations-, noch einem Mehrheitsprinzip verpflichtet. Wie die Überlegungen zum Massen- und Organisationsbegriff verdeutlicht haben dürften, ist die Demokratisierung des Diskurses nicht einfach durch eine ansteigende Zahl der PhilologInnen zu leisten. Vielmehr geht es bei dem Frühromantiker wie bei den Vertretern der Dekonstruktion um die Art der Arbeit selbst. So versteht sich Paul de Mans Plädoyer für kleine Forschergruppen, ${ }^{102}$ das - wie eingangs gesagt - keine terroristische Zellenbildung anstoßen, aber auch nicht als elitäre Geste erscheinen will, die andere ausschließt. Diese kleinen Forschergruppen stellen überdies keine Repräsentanten dar, die in Stellvertretung anderer entscheiden. Wenn der epideiktische Redner oder Dichter überhaupt etwas srepräsentiertı, dann den Anlass seines Diskurses. Analoges gilt für die Schlegel'schen PhilologInnen: Sie lassen sich selbst durch den Anlass ihrer Arbeit affizieren und stellen damit (selbst) diesen Anlass für andere dar. Und zwar in einem Kreis, der ebenso wie derjenige der epideixis institutionell nicht zu schließen ist, sondern sich in eine Unzahl von (post-souveränen) Kreisen zersetzen kann. Sowohl die Selbstaffektion als auch die Zersetzung birgt Chancen und Gefahren für die Philologie.

IO2 Siehe hierzu Paul de Man:»Field of Comparative Literature. Analysis of Needs (1967)«. In: The Paul de Man Notebooks. Hg. von Martin McQuillan. Edinburgh 20I4, S. 229-235, hier S. 235. 